\title{
LES DÉTERMINANTS DU DÉVELOPPEMENT DE L'AUTO-EFFICACITÉ ENTREPRENEURIALE DANS UN CONTEXTE DE MENTORAT ${ }^{1}$
}

\author{
Etienne St-Jean, professeur \\ Département des Sciences de la Gestion \\ Institut de Recherche sur les PME \\ Université du Québec à Trois-Rivières \\ 3351, boul. des Forges, CP 500 \\ Trois-Rivières, Québec G9A 5H7 \\ Tél. : (819) 376-5011 poste 4293 \\ Fax : (819) 376-5138 \\ Courriel : etienne.st-jean@uqtr.ca \\ Cynthia Mathieu, professeure \\ Département des Sciences de la Gestion \\ Institut de Recherche sur les PME \\ Université du Québec à Trois-Rivières \\ 3351, boul. des Forges, CP 500 \\ Trois-Rivières, Québec G9A $5 \mathrm{H} 7$ \\ Tél. : (819) 376-5011 poste 3165 \\ Courriel : cynthia.mathieu@uqtr.ca
}

\section{RÉSUMÉ}

Par le passé, des recherches qualitatives ont fait ressortir l'importance du mentorat dans le développement de l'auto-efficacité entrepreneuriale des novices. Également, l'orientation dans un but d'apprentissage est reconnue comme une disposition individuelle exerçant une influence positive dans le développement du sentiment d'auto-efficacité Cette recherche propose de vérifier si le sentiment d'auto-efficacité peut être développé chez l'entrepreneur novice lorsqu'il est jumelé avec un mentor en tenant compte de l'orientation dans un but d'apprentissage. Pour y parvenir, 360 entrepreneurs mentorés du réseau de mentorat d'affaires de la Fondation de l'entrepreneurship ont été interrogés. En utilisant un modèle d'équations structurelles, il est apparu que trois des six sous-échelles d'auto-efficacité entrepreneuriale sont développées en fonction du mentorat reçu. L'orientation dans un but d'apprentissage du mentoré influence à la fois le développement de l'auto-efficacité ainsi que le niveau de mentorat reçu. Ces résultats confirment que le mentor influence cette dimension affective chez l'entrepreneur novice et laissent présager le rôle important que pourrait jouer l'orientation dans un but d'apprentissage des entrepreneurs dans leur cheminement de carrière.

\footnotetext{
${ }^{1}$ Les auteurs tiennent à remercier le Conseil de Recherche en Sciences Humaines du Canada pour leur soutien à cette recherche.

St-Jean, É. et C. Mathieu (2011), « Les déterminants de l'auto-efficacité entrepreneuriale dans un contexte de mentorat », Revue de l'entrepreneuriat, Vol. 10, no. 3, p. 13-32.
} 


\section{INTRODUCTION}

Depuis quelques années, on assiste à la mise en place de programmes destinés à soutenir les entrepreneurs novices dans les années suivant le démarrage de leur entreprise. L'une des formules proposée consiste à jumeler un entrepreneur novice avec un entrepreneur expérimenté, ce dernier lui offre des conseils et des pistes de réflexion visant à lui éviter des erreurs coûteuses, voire fatales (St-Jean et Audet, Under press; Sullivan, 2000). À titre d'exemple, le programme SCORE ${ }^{2}$ aux États-Unis, fondé dans les années 1970 et financé par la Small Business Administration (SBA), a soutenu plus de 8 millions de dirigeants de petites entreprises grâce à son réseau de plus de 12000 mentors bénévoles. En Europe, d'autres initiatives similaires existent telles que celle soutenue par le Business Link d'Angleterre, le programme Mentor Eget Företag de Suède ou France Initiative (en France), avec près de 5000 mentors bénévoles, pour ne nommer que ces programmes-là.

L'un des objectifs de ces programmes est de pallier au manque de compétence et d'expérience des nouveaux entrepreneurs, un des principaux problèmes causant la faillite des entreprises naissantes, ce qui se manifeste bien souvent par un manque de vision et par une difficulté, voire une incapacité, de trouver un créneau rentable à la nouvelle entreprise (Baldwin et al., 1997; Festervand et Forrest, 1991; Gaskill et al., 1993). Dans la documentation scientifique, plusieurs témoignages d'entrepreneurs novices ayant reçu l'aide d'un mentor viennent suggérer que cette forme d'accompagnement leur serait utile, notamment au niveau de leur développement professionnel (Cull, 2006; Wikholm et al., 2005). Toutefois, aucune recherche n'a démontré auprès d'un échantillon représentatif suffisamment grand l'effet du mentorat pour un entrepreneur novice. Cette recherche vise à combler cette lacune, en ciblant particulièrement l'auto-efficacité comme étant l'une des retombées importantes du mentorat d'entrepreneur (Nandram, 2003; St-Jean, 2008). L'objectif est de vérifier si le mentorat contribue à développer le sentiment d'auto-efficacité auprès des entrepreneurs novices.

Pour y parvenir, nous présenterons la littérature qui aborde le mentorat comme forme d'accompagnement. Par la suite, nous verrons en quoi le mentorat est susceptible de développer l'auto-efficacité entrepreneuriale et comment l'orientation dans un but d'apprentissage du novice est susceptible d'influencer cette relation. Tout de suite après, nous présenterons la méthodologie utilisée et les résultats obtenus, avant de les commenter et de conclure sur les implications de ces résultats et les recherches envisagées dans le futur.

\section{EXAMEN DE LA DOCUMENTATION}

Dans cette section, il sera d'abord question du mentorat comme forme d'accompagnement, avec l'objectif de le distinguer de d'autres postures pouvant être adoptées par des accompagnateurs. Par la suite, nous plongerons directement dans les concepts relatifs au mentorat, en débutant par les fonctions du mentor, considérés comme étant une mesure du mentorat reçu, avant d'aborder l'auto-efficacité comme étant l'une retombée tout à fait probable d'une telle relation et de conclure avec l'effet de l'orientation dans un but d'apprendre de la personne accompagnée et de son effet à la fois sur le mentorat mais également pour soutenir le développement de l'autoefficacité.

\subsection{LE MENTORAT COMME FORME D’ACCOMPAGNEMENT}

Bien que l'on assiste depuis quelques années à une multiplication des programmes de mentorat, ce phénomène n'est pas nouveau. Le mot «mentor» provient d'ailleurs de L'Odyssée

\footnotetext{
${ }^{2}$ Acronyme pour Service Corps of Retired Executives. Information au www.score.org.
} 
d'Homère, dont Ulysse, le héros, confia son fils Télémaque à son bon ami Mentor pendant son voyage. Mentor devait s'occuper de l'éducation de Télémaque ainsi que du développement de son identité dans le monde adulte. Lorsque Mentor s'adressait à Télémaque, la déesse Athéna parlait au travers de lui. Il accédait alors à des qualités divines et devenait l'incarnation de la sagesse. Dans notre monde contemporain et en s'inspirant de la métaphore grecque, un mentor est généralement une personne possédant certaines qualités ou qui est en position d'autorité et qui veille de façon bienveillante sur un individu plus jeune, lequel bénéficie des conseils et du soutien de son mentor. Dans le contexte de l'entrepreneur, bien qu'il puisse exister plusieurs définitions, le mentorat concerne l'établissement d'une relation de soutien entre un entrepreneur novice (nommé le mentoré) et un entrepreneur d'expérience (appelé le mentor), le dernier permettant au premier de se développer en tant que personne. Tel que le suggère Paul (2004), le mentorat se distinguerait du coaching, du tutorat ou du compagnonnage du fait qu'il est davantage orienté vers la quête de sens plutôt que l'acquisition de techniques. Tout en étant ancré davantage vers l'action, contrairement au counselling, au conseil ou à la médiation, il se distinguerait quand même du parrainage, en étant légèrement moins orienté vers l'action que cette forme d'accompagnement. Ainsi, l'idée sous-jacente au mentorat serait l'éducation, où le mentor est dans un registre de " conduite » et de " guidage ", et se distingue du coaching, basé sur l'idée d'entraînement, ou du counselling, axé sur une relation d'aide psychologique (Deschamps et al., 2010). Évidemment, il faut souligner que les différentes formes d'accompagnement finissent par se recouper dans la pratique, d'une part, et qu'on en recense néanmoins 13 formes distinctes, d'autre part (D'abate et al., 2003). Soulignons également que le mentor place les intérêts de son mentoré comme une priorité absolue, et non comme faisant partie d'un ensemble de priorités (Gibson, 2005). Ainsi, pour reconnaître une relation de mentorat, tel que le proposent Haggard et al. (2011), trois dimensions doivent être présentes : il doit y avoir une relation de réciprocité ${ }^{3}$, possédant des bénéfices développementaux pour l'accompagné, en particulier pour la carrière de celui-ci, ainsi que des interactions régulières et substantielles dans une perspective à long terme.

\subsection{LES FONCTIONS DU MENTOR ET LE MENTORAT REÇU}

Dans les écrits qui concernent le mentorat tel qu'il se pratique dans la grande organisation, les fonctions du mentor font référence aux différents rôles joués par le mentor dans la relation de mentorat (Kram, 1985). Dans une recension exhaustive de la littérature sur le mentorat en contexte organisationnel, on mentionne que c'est grâce aux fonctions du mentor que le mentoré développera des retombées de sa relation (Wanberg et al., 2003). Voilà pourquoi ces auteurs considèrent les fonctions du mentor comme une mesure du mentorat reçu ou transmis. Les différentes fonctions du mentor étudiées dans la grande organisation se regroupent généralement en trois grandes catégories: les fonctions psychologiques, celles relatives à la carrière et la fonction de modèle de rôle (Bouquillon et al., 2005; Pellegrini et Scandura, 2005; Scandura et Williams, 2001). Dans un contexte d'entrepreneur, bien que la nature des fonctions soit différente, on confirme l'existence de trois méta-fonctions distinctes, soit les fonctions qui concernent la carrière, celles ne nature psychologique et la fonction de modèle de rôle (St-Jean, 2010). En somme, il est tout probable que les fonctions du mentor, que l'on considère comme une mesure de mentorat reçu, contribuent à développer certaines retombées chez l'entrepreneur novice, mais l'état des connaissances ne permet pas de le démontrer.

\subsection{MENTORAT ET AUTO-EFFICACITÉ ENTREPRENEURIALE}

Cela étant dit, dans un contexte d'entrepreneur, peu de choses dépassant l'anecdote évidente sont connues, tel que le rappelait Ensher et al. (2000). Malgré cela, il y a bien sûr des chercheurs

\footnotetext{
${ }^{3}$ Qui reprend les principes de parité de Paul (2004)
} 
qui ont investigué des relations de mentorat d'entrepreneur. Par exemple, quelques-uns ont effectué un recensement systématique des retombées du mentorat pour l'entrepreneur (Deakins et al., 1998; St-Jean et al., 2007). D'autres ont présenté des études descriptives d'entrepreneurs ayant reçu les services d'un "mentor » (Bisk, 2002; McGregor et Tweed, 2002; Nandram, 2003; Wikholm et al., 2005) ou une étude de quelques cas de mentorés (Cull, 2006; Gravells, 2006). Malgré les nombreux témoignages d'entrepreneurs mentorés qui suggèrent que le mentorat leur a été bénéfique, l'effet de cette forme d'accompagnement dans leur vie n'a pas été démontré auprès d'un échantillon représentatif dans aucune de ces recherches. La seule recherche empirique réalisée avec un tel échantillon est celle de Waters et al. (2002), qui démontre l'effet du mentorat sur l'estime de soi et le succès perçu du mentoré. Il faut cependant souligner que les mentors du programme étudié avaient reçu des directives précises quant aux rôles qu'ils devaient jouer et avaient été sélectionnés pour leurs compétences spécifiques, suggérant que les relations pourraient être davantage considérées comme du coaching, plutôt que du mentorat. Bref, l'état actuel des connaissances permet difficilement de se prononcer quant à la contribution réelle du mentorat dans le cheminement entrepreneurial.

Toutefois, selon Krueger (2000), le mentor de l'entrepreneur serait utile dans le développement de l'auto-efficacité de ce dernier. Le sentiment d'auto-efficacité fait référence à la croyance d'un individu en sa capacité d'accomplir les tâches spécifiques qu'il entreprend (Bandura, 1997). La perception d'auto-efficacité ne concerne pas le nombre d'habiletés qu'un individu possède mais la croyance de ce qu'il peut faire avec les habiletés qu'il possède dans une variété de circonstances. Selon la théorie sociocognitive de Bandura (1986), quatre processus influencent le développement de l'auto-efficacité chez un individu: la maîtrise personnelle, l'apprentissage social ou modelant, la persuasion par autrui et l'état physiologique et émotionnel perçu, tel que l'excitation ou l'anxiété. Le mentorat intervient principalement au niveau de l'apprentissage social et de la persuasion par autrui. Il faut également considérer que l'autoefficacité des mentorés pourrait être stimulée ou influencée par d'autres facteurs, tels son sexe (ex. Wilson et al., 2007), son âge (ex. Maurer, 2001), sa scolarité (ex. Zimmerman et MartinezPons, 1990) ou ses expériences précédentes. Par ailleurs, étant dans l'action en tant que dirigeant de sa PME et suivant les conseils de son mentor, ses expériences en temps réel pourraient stimuler son auto-efficacité par le biais de la maîtrise personnelle qu'il va développer. Ainsi, le mentorat pourrait être à même de stimuler le mentoré par le biais des trois principaux processus de développement de l'auto-efficacité (Bandura, 1986)

Les individus qui croient en leurs capacités vont affronter les tâches difficiles comme des défis à relever, plutôt que des menaces à éviter. Chez les gestionnaires, par exemple, l'auto-efficacité est reliée positivement à l'évaluation de leur performance (Robertson et Sadri, 1993). Comme le suggère Johannisson (1991), la présence de mentors ou de modèles de rôle pourrait influencer le sentiment d'auto-efficacité. Par exemple, on note que le rôle des parents, comme modèles d'entrepreneurs, influence positivement le niveau d'auto-efficacité spécifique à l'entrepreneur (Scherer et al., 1989). Lors de sa relation, un mentoré pourrait se voir attribuer des tâches ou des défis à relever par son mentor afin d'augmenter sa compétence (Cull, 2006; Kim, 2007). Un entrepreneur ayant un haut niveau d'auto-efficacité serait alors plus enclin à croire en sa capacité à relever le défi ou les tâches proposées par son mentor et, de ce fait, à les réaliser, produisant davantage de retombées. D'ailleurs, on observe une relation significative entre le sentiment d'auto-efficacité et le fait d'avoir un mentor lorsqu'on est en affaires (Nandram, 2003; Ozgen et Baron, 2007). Ces constats suggèrent l'hypothèse suivante :

Hypothèse 1 : Le niveau de mentorat reçu influence positivement le sentiment d'autoefficacité entrepreneuriale. 


\subsection{L'ORIENTATION DANS UN BUT D'APPRENTISSAGE}

Les individus orientés dans un but d'apprentissage souhaitent apprendre de nouvelles choses et augmenter leurs compétences dans certaines activités (Button et al., 1996). L'orientation dans un but d'apprentissage est une disposition psychologique assez stable que les individus amènent dans leurs relations avec les autres. Cette orientation stimule le comportement et influence l'interprétation et la réaction à certaines retombées (Dweck, 1986). Un individu avec une orientation dans un but d'apprentissage élevée sera plus enclin à considérer ses compétences comme malléables et ainsi, aborder les tâches avec l'intention de les développer. D'ailleurs, il faut souligner que les individus croyant que leur intelligence est « fixe » vont avoir un sentiment d'auto-efficacité plus faible que ceux qui la croient malléable (Kanfer, 1990). En outre, l'effet de l'orientation dans un but d'apprentissage sur l'auto-efficacité a été démontré dans des recherches précédentes (Bell et Kozlowski, 2002; Phillips et Gully, 1997). Ces considérations amènent l'hypothèse suivante :

Hypothèses 2: L'orientation dans un but d'apprentissage influence positivement le sentiment d'auto-efficacité entrepreneuriale.

De plus, l'orientation dans un but d'apprentissage semble influencer autant les fonctions du mentor que les retombées de la relation (Egan, 2005; Godshalk et Sosik, 2003). Cela laisse présager que cette disposition psychologique chez le mentoré aurait également une influence auprès du mentor. Il est probable qu'un mentoré orienté dans un but d'apprentissage profiterait davantage de l'opportunité d'apprentissage offerte par sa relation de mentorat, stimulant le mentor à exercer ses fonctions avec plus d'intensité (Kim, 2007). Il est également possible qu'un mentoré orienté dans un but d'apprentissage perçoive de manière plus marquée les fonctions exercées par son mentor, sachant que celle-ci sont la principale source d'apprentissage du mentoré (Lankau et Scandura, 2002; Wanberg et al., 2003). Conséquemment, cela amène l'hypothèse suivante :

Hypothèse 3: L'orientation dans un but d'apprentissage influence positivement le niveau de mentorat reçu.

Afin de vérifier ces hypothèses, une méthodologie sera proposée dans la section qui suit pour tester le modèle représenté par la Figure 1.

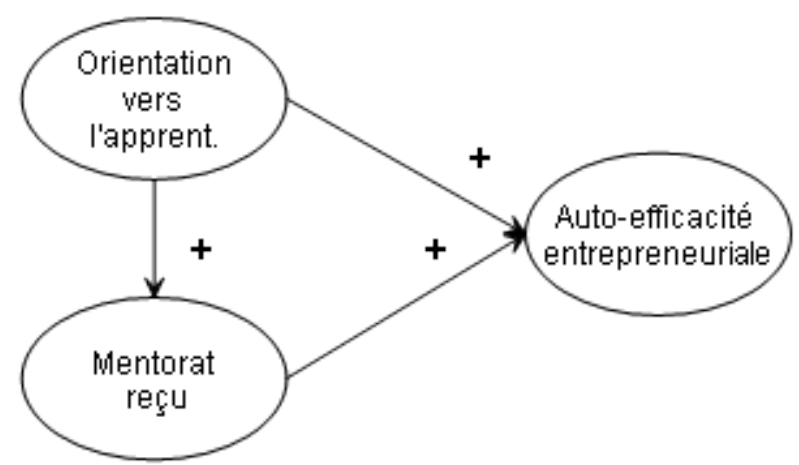

Figure 1. Modèle conceptuel de l'effet des fonctions du mentor et de l'orientation dans un but d'apprentissage sur le sentiment d'auto-efficacité entrepreneuriale.

\section{MÉTHOdOLOGIE}


Dans la présente section, nous présenterons le programme dans lequel la recherche s'est déroulée, avant de présenter l'échantillon sélectionné, les différentes mesures ainsi que la méthode utilisée pour répondre aux hypothèses soulevées précédemment.

\subsection{LE PROGRAMME ÉTUDIÉ}

Le réseau de mentorat d'affaires, qui sert de toile de fond à la présente recherche, a été créé en 2000 par la Fondation de l'entrepreneurship, un organisme voué au développement économique sur le territoire du Québec. Il est offert aux entrepreneurs novices par le biais d'environs 70 cellules de mentorat dispersées sur l'ensemble du territoire du Québec. Ces cellules sont généralement soutenues par des organismes de développement économique tels les Centres locaux de développement (CLD), les Sociétés d'aide au développement des collectivités (SADC) et les chambres de commerce locales. Ces organismes assurent le développement du programme au niveau local ou régional, tout en adhérant au modèle du mentorat d'affaires développé par la Fondation. De façon plus précise, les organismes locaux se dotent d'un coordonnateur de la cellule, lequel est responsable du recrutement des mentors, de l'organisation des séances de formation à leur intention, de la promotion du programme auprès des entrepreneurs novices, du jumelage des participants et de la supervision de la relation qui s'ensuit. Les entrepreneurs novices peuvent bénéficier du soutien d'un mentor pour un coût minime, soit quelques centaines de dollars annuellement, et parfois même gratuitement. Afin de bien encadrer le développement local, la Fondation de l'entrepreneurship développe des ateliers de perfectionnement orientés sur la relation mentor-mentoré pour éclairer concrètement le rôle des mentors auprès des nouveaux entrepreneurs. Basé sur un code d'éthique d'intervention où la confidentialité des relations est d'une importance capitale, le service de mentorat d'affaires s'est également doté d'un contrat-type pour encadrer et guider les parties dans le choix des modalités de leur relation et des objectifs à atteindre. Il s'agit donc d'un mentorat de type « formel ».

\section{2 ÉCHANTILLON RETENU POUR L'ANALYSE}

La population étudiée représente l'ensemble des entrepreneurs mentorés du réseau de mentorat d'affaires de la Fondation de l'entrepreneurship, qui ont eu au moins trois (3) rencontres avec leur mentor, ou qui sont toujours en relation avec lui, et dont l'adresse courriel était valide, ce qui représente 981 personnes. Les mentorés ont été contactés par courriel pour participer à la recherche et deux relances ont été effectuées auprès des non-répondants, ce qui a permis d'obtenir 362 répondants, pour un taux de réponse de $36,9 \%$. Puisqu'un portrait de la population n'était pas disponible a priori, une comparaison entre les répondants hâtifs (répondants dès la première sollicitation) et tardifs (après les relances) a été faite, tel que suggéré par Armstrong et Overton (1977). Il n'existe aucune différence significative entre les variables démographiques, celles relatives à l'entreprise et les variables mesurées dans cette recherche, ce qui suggère que l'échantillon corresponde bien à la population étudiée.

L'échantillon contient 162 hommes $(51,6 \%)$ et 152 femmes $(48,4 \%)$. Ils ont été jumelés à 275 mentors hommes $(81,4 \%)$ et à 63 mentors femmes $(18,6 \%)$. Cette situation est cependant «normale », en considérant la plus grande représentation des hommes parmi les mentors disponibles, sans doute à cause d'une réalité historique : il y avait moins de femmes en affaires dans les 40 dernières années qu'aujourd'hui. Conséquemment, le bassin potentiel de mentors femmes est davantage restreint que pour celui des hommes. Les mentorés sont assez scolarisés puisque $173(55 \%)$ d'entre eux ont au moins un diplôme universitaire. Ils ont 39,8 ans en moyenne (écart-type de 8,97, médiane de 38 ans) et l'âge varie entre 23 et 70 . Au moment du démarrage, $24 \%$ n'avaient aucune expérience du secteur d'activité de leur entreprise, 33,2\% moins d'un an, 46,2\% moins de trois ans et $61,6 \%$ moins de cinq ans. Au niveau de l'expérience en affaires au démarrage, la majorité $(51,1 \%)$ n'avaient aucune expérience, 63,4\% avaient 
moins d'un an, 73,6\% avaient moins de trois ans et $82,9 \%$ avaient moins de cinq ans. La presque totalité des mentorés avaient une entreprise active au moment du jumelage (293 sur $314,93,3 \%$ ) et les autres étaient en processus de démarrage. Celle-ci avait peu d'employés, avec une moyenne de 4,48 (écart-type 9,69, médiane 2). Le chiffre d'affaires est majoritairement inférieur à $100000 \$ C A D$ annuellement $(62,8 \%), 88,9 \%$ ayant moins de $500000 \$$ de c.a. par an et pour seulement $8,6 \%$, il était supérieur à 1 million $\$$. Au niveau des profits bruts, incluant les salaires et bonis du dirigeant principal, la situation n'est guère plus reluisante. La très grande majorité $(68,1 \%)$ déclarent avoir des profits inférieurs à $25000 \$$ annuellement, $83,5 \%$ font moins de $50000 \$$ et seulement $6,3 \%$ font plus de $100000 \$$. Les secteurs industriels sont variés, mais avec une légère concentration dans les services professionnels $(62$, pour 23,0\%), dans la fabrication (39, pour $14,4 \%)$ et dans le commerce de détail (32, pour 11,9\%). Les relations de mentorat ont duré en moyenne 16,07 mois (écart-type de 14,4, médiane de 13). Les rencontres avec le mentor duraient en moyenne 68,52 minutes (écart-type de 14,4, médiane de 67) et il y avait un peu moins d'une rencontre par mois $(0,807)$, la médiane étant d'une rencontre par mois. La majorité des répondants étaient encore en relation de mentorat au moment de répondre $(58,6 \%)$.

\subsection{MESURES}

\subsubsection{Orientation dans un but d'apprentissage}

La mesure utilisée est celle développée par Button et al. (1996) en 8 items, lesquels sont enregistrés sur une échelle Likert 7 allant de 1, «Fortement en désaccord » à 7, « Fortement en accord». Les items vérifient l'attitude du mentoré à rechercher des situations où il peut apprendre : 1-Avoir l'opportunité d'accomplir un travail qui me permet de relever un défi est important pour moi, 2-Lorsque je n'arrive pas à accomplir une tâche difficile, ça m'incite à y travailler encore plus fort la fois suivante, 3-Je préfère travailler sur des tâches qui me forcent à apprendre de nouvelles choses, 4-Avoir l'opportunité d'apprendre de nouvelles choses est important pour moi, 5-Je donne le meilleur de moi-même lorsque je travaille sur une tâche passablement difficile, 6-Je m'efforce d'améliorer mes performances passées, 7-Avoir l'occasion de développer de nouvelles habiletés est important pour moi et 8-Lorsque j'ai de la difficulté à résoudre un problème, j'aime essayer différentes approches pour voir laquelle va fonctionner ${ }^{4}$. D'autres recherches sur le mentorat ont utilisé cette mesure avec de bons résultats quant à l'unidimensionnalité et à la cohérence interne (Godshalk et Sosik, 2003). L'analyse confirmatoire avec LISREL, un logiciel spécialisé dans ce type d'analyse, indique que tous les items sont significatifs pour expliquer la variable latente. La corrélation des erreurs de mesure a été permise entre trois paires, soit LGO5 et LGO6, LGO5 et LGO7 ainsi que LGO7 et LGO8. Les indices d'ajustement du modèle confirmatoire sont excellents, avec un $\chi^{2}$ de 23,0012 pour 17 degrés de liberté $(\mathrm{p}=0,1492)$, le RMSEA étant de 0,03721 , le SRMR à 0,03492 , le CFI de 0,9979 et un NFI de 0,9921. Quant à l'alpha Cronbach, il est de 0,927. Le construit donc jugé acceptable pour l'analyse subséquente.

\subsubsection{Fonctions du mentor}

La mesure utilisée a été développée pour cette recherche. Elle représente le niveau de mentorat reçu par le mentoré. Dans un premier temps, les commentaires de 51 mentorés répartis dans six groupes de discussion ainsi que d'un groupe de huit mentors ont été analysés afin d'identifier les différentes fonctions jouées par un mentor. Une proposition conceptuelle comprenant neuf fonctions a été rédigée et corrigée par deux chercheurs ainsi que les 35 items censés les mesurer. La proposition conceptuelle a été soumise à trois experts qui devaient, dans un premier temps, classer les 35 items dans les neuf fonctions proposées et, par la suite, une fois que le bon

\footnotetext{
${ }^{4}$ Ces items correspondent à LGO1 jusqu'à LGO8, dans l'ordre.
} 
classement leur a été fourni, ils devaient porter un regard critique sur la proposition, en suggérant des nouvelles fonctions ou des retranchements, des nouvelles formulations d'items, et ainsi de suite. Le premier des experts fait partie des dirigeants du réseau de mentorat d'affaires. Le deuxième est un professeur d'université retraité dans les domaines de l'éducation et de l'entrepreneuriat et qui est mentor dans le réseau étudié. Le dernier est aussi mentor et consultant-formateur, notamment sur les différents rôles du mentor, pour le compte du réseau étudié. Les experts ont obtenu $85,3 \%, 82,4 \%$ et $79,4 \%$ de réponses correctes à l'exercice de classement des items $(\mathrm{M}=82,4 \%)$ et la plupart des commentaires formulés n'étaient pas substantiels et ont été considérés dans la reformulation de la proposition théorique. Aucune nouvelle fonction n'a été suggérée et aucun expert n'a souligné le manque de pertinence de l'une d'elles.

Dans un deuxième temps, cette proposition a été soumise à l'analyse quantitative auprès de l'échantillon de cette recherche. À noter que les fonctions qui sont ressorties se regroupent en des fonctions psychologiques (réflecteur, sécurisation, motivation et confident), d'autres reliées à la carrière (intégration, soutien informationnel, guide et confrontation) et finalement de modèle de rôle (modèle), allant dans le même sens que les regroupements proposés précédemment dans d'autres contextes (Bouquillon et al., 2005; Tepper et al., 1996). L'analyse confirmatoire avec LISREL a permis de mettre en lumière que tous les items sont significatifs pour expliquer les neuf fonctions du mentor (testées dans trois regroupements de fonctions) et qu'autant les principaux indices d'adéquation que l'indice de consistance interne (Alpha Cronbach) étaient acceptables ${ }^{5}$. Dans le cadre de cette recherche, par souci de parcimonie, un modèle reprenant l'item le plus significatif de chacune des fonctions du mentor sera utilisé (voir Tableau 1). Dans la plupart des cas, cet item représente assez bien l'idée générale de la fonction proposée.

Tableau 1. Outil de mesure parcimonieux des fonctions du mentor

\begin{tabular}{ll}
\hline Item & Libellé \\
\hline Réflecteur & Il me permet de me faire une image précise de moi et de mon entreprise \\
Sécurisation & Il me sécurise \\
Motivation & Il croit que je peux réussir comme entrepreneur \\
Confident & Je le considère comme un ami \\
Intégration & Il me met en relation avec des gens qu'il connaît \\
Soutien & Il me fournit des informations et des renseignements reliés au monde des \\
informationnel & affaires \\
Confrontation & Il n'hésiterait pas à me contredire s'il n'était pas d'accord \\
Guide & Il me propose d'autres points de vue \\
Modèle & Il m'expose ses réussites et ses échecs \\
\hline
\end{tabular}

L'analyse confirmatoire de cette mesure des fonctions du mentor avec LISREL a démontré que tous les items sont significatifs à $\mathrm{p} \leq 0,01$ pour expliquer la variable latente. De plus, le $\chi^{2}$ est 36,29 pour 27 degrés de liberté $(\mathrm{p}=0,10908)$, le RMSEA est à 0,04667 , le SRMR est à 0,03780 et le CFI est à 0,9959 , ce qui indique un excellent modèle. Le coefficient $\alpha$ pour les neuf items est de 0,898, ce qui dépasse aussi les seuils acceptables (Tabachnick et Fidell, 2007). Pour les analyses subséquentes, ces neuf items vont donc converger vers une variable latente représentant les fonctions du mentor.

\footnotetext{
${ }^{5}$ Les résultats obtenus sont disponibles sur demande et ont été exclus de la présentation par souci d'économie d'espace.
} 


\subsubsection{Sentiment d'auto-efficacité entrepreneuriale}

La meilleure façon de mesurer ce construit est de combiner les sous-échelles développées par De Noble et al. (1999) et Anna et al. (2000). Elles sont complémentaires en ce qu'elles touchent à des facettes différentes du construit, à l'exception de la reconnaissance d'opportunités, qui a été couverte par les deux groupes d'auteurs. Ces mesures ont d'ailleurs été utilisées subséquemment dans d'autres recherches de façon partielle (voir Barbosa et al. (2007)) ou de façon complète pour De Noble et al. (1999) (voir Hmielski et Corbett (2008)). Seule la facette qui concerne la capacité à initier des relations avec les investisseurs semble ici moins pertinente, en considérant que la levée de capitaux n'est pas tout à fait similaire au Québec et aux ÉtatsUnis. Elle a donc été retranchée. Le Tableau 2 résume les mesures choisies.

Tableau 2. Les mesures d'auto-efficacité entrepreneuriale sélectionnées

\begin{tabular}{|c|c|c|c|}
\hline $\begin{array}{l}\text { ESE Subscale - } \\
\text { Defining Core } \\
\text { Purpose (DCP) }\end{array}$ & $\begin{array}{l}\text { De Noble, } \\
\text { Jung, et } \\
\text { Ehrlich } \\
\text { (1999) }\end{array}$ & $\begin{array}{l}\text { 1-Je peux énoncer la vision que j'ai de mon } \\
\text { entreprise et de mes valeurs, 2-Je peux inspirer les } \\
\text { autres à endosser ma vision de mon entreprise et } \\
\text { mes valeurs et 3-Je peux développer un plan } \\
\text { d'action pour poursuivre une opportunité }\end{array}$ & $\begin{array}{l}\% \operatorname{Var} \\
76,47 \\
\alpha 0,841\end{array}$ \\
\hline $\begin{array}{l}\text { ESE Subscale - } \\
\text { Coping w. } \\
\text { Unexpec. Chal. } \\
\text { (CUC) }\end{array}$ & $\begin{array}{l}\text { De Noble, } \\
\text { Jung, et } \\
\text { Ehrlich } \\
\text { (1999) }\end{array}$ & $\begin{array}{l}\text { 1-Je peux demeurer productif sous le stress continu, } \\
\text { la pression et les conflits, 2-Je peux tolérer des } \\
\text { changements inattendus dans l'environnement } \\
\text { d'affaires et 3-Je peux persévérer face à l'adversité }\end{array}$ & $\begin{array}{l}\% \operatorname{Var} \\
68,93 \\
\alpha 0,772\end{array}$ \\
\hline $\begin{array}{l}\text { ESE Subscale - } \\
\text { Opportunity } \\
\text { Recognition (OR) }\end{array}$ & $\begin{array}{l}\text { Anna et } \\
\text { al. }(2000)\end{array}$ & $\begin{array}{l}\text { 1-Je peux percevoir les besoins non comblés dans le } \\
\text { marché, } 2 \text {-Je peux reconnaître les produits voués au } \\
\text { succès et } 3 \text {-Je peux discerner les opportunités }\end{array}$ & $\begin{array}{l}\% \operatorname{Var} \\
81,07 \\
\alpha 0,882\end{array}$ \\
\hline $\begin{array}{l}\text { ESE Subscale - } \\
\text { Formal Planning } \\
\text { (FP) }\end{array}$ & $\begin{array}{l}\text { Anna et } \\
\text { al. }(2000)\end{array}$ & $\begin{array}{l}\text { 1-Je peux écrire des plans formels, } 2 \text {-Je peux } \\
\text { traduire ma vision en stratégie et } 3 \text {-Je peux } \\
\text { communiquer mes objectifs par écrit }\end{array}$ & $\begin{array}{l}\% \operatorname{Var} \\
75,88 \\
\alpha 0,841\end{array}$ \\
\hline $\begin{array}{l}\text { ESE Subscale - } \\
\text { Economic } \\
\text { Management (EM) }\end{array}$ & $\begin{array}{l}\text { Anna et } \\
\text { al. }(2000)\end{array}$ & $\begin{array}{l}\text { 1-Je peux gérer les dépenses, 2-Je peux contrôler } \\
\text { les coûts d'opération de mon entreprise et } 3 \text {-Je peux } \\
\text { gérer les entrées et sorties de fonds }\end{array}$ & $\begin{array}{l}\% \operatorname{Var} \\
91,83 \\
\alpha 0,955\end{array}$ \\
\hline $\begin{array}{l}\text { ESE Subscale } \\
\text { Human/concept.Co } \\
\text { mpet. (HCC) }\end{array}$ & $\begin{array}{l}\text { Anna et } \\
\text { al. }(2000)\end{array}$ & $\begin{array}{l}\text { 1-Je peux superviser, influencer et diriger, } 2-\mathrm{Je} \\
\text { peux organiser et motiver les autres, et } 3 \text {-Je peux } \\
\text { faire rouler mon entreprise sans problème }\end{array}$ & $\begin{array}{l}\text { \%Var } \\
79,47 \\
\alpha 0,860\end{array}$ \\
\hline
\end{tabular}

Ces mesures ont été testées au niveau de leur unidimensionnalité par une analyse factorielle exploratoire et leur alpha de Cronbach $(\alpha)$ a été calculé. Toutes les mesures sont adéquates pour l'analyse subséquente car elles sont toutes unidimensionnelles et possèdent des alphas supérieurs aux normes proposées $(>0,70)$.

\subsection{MÉTHODE}

Un modèle d'équations structurelles utilisant LISREL a été testé pour chacune des mesures d'auto-efficacité, selon le modèle illustré à la Figure 1. Plutôt que d'utiliser des variables latentes amalgamées, les items ont été directement entrés dans le modèle pour créer un modèle d'équations structurelles de deuxième niveau. La matrice de corrélations polychoriques a été calculée et utilisée pour tester le modèle, ce qui permet de réduire les biais d'estimation, d'autant plus qu'elle n'est pas sensible à la forme que prend la distribution marginale (Jöreskog et Sörbom, 2002; Tabachnick et Fidell, 2007). Les non-répondants à au moins un item ont été retirés de l'analyse, cette procédure permettant d'améliorer la qualité de la matrice, conservant alors 254 sujets pour l'analyse (Jöreskog et Sörbom, 2002). Pour une force statistique de 0,80 , à 160 degrés de liberté, moins de 160 sujets sont nécessaires (MacCallum et al., 1996). En 
conséquence, le nombre de répondants actuel procure une force statistique suffisamment élevée pour permettre l'utilisation de ce test.

\section{RÉSULTATS}

Le Tableau 3 présente les moyennes, écarts-type et corrélations entre les construits ${ }^{6}$. Au niveau du modèle structurel testé, toutes les relations entre les variables manifestes et les variables latentes sont significatives à $\mathrm{p} \leq 0,001$. Afin d'améliorer la qualité du modèle global, sept corrélations entre les erreurs de mesure d'un même construit ont été permises ${ }^{7}$. Il est logique de penser que ces erreurs de mesure puissent corréler entre elles, étant donné qu'elles appartiennent au même construit, d'une part, et que la méthode employée pour mesurer le concept est identique pour chacun des items (des échelles Likert), d'autre part.

Tableau 3. Moyennes, écarts-type et corrélations des variables

\begin{tabular}{lccccccccc}
\hline Variable & Moy. & E.-T. & 1 & 2 & 3 & 4 & 5 & 6 & 7 \\
\hline 1-LGO & 6.24 & 0.88 & & & & & & & \\
2-Fonctions & 5.39 & 1.15 & .01 & & & & & & \\
3-ESE-DCP & 6.17 & 0.84 & $.29^{* * *}$ & $.15^{*}$ & & & & & \\
4-ESE-CUC & 5.99 & 0.86 & $.31^{* * *}$ & .06 & $.54^{* * *}$ & & & & \\
5-ESE-OR & 5.75 & 1.00 & $.23^{* * *}$ & $.15^{*}$ & $.53^{* * *}$ & $.51^{* * *}$ & & & \\
6-ESE-FP & 5.68 & 1.03 & $.20^{* * *}$ & $.12^{*}$ & $.54^{* * *}$ & $.46^{* * *}$ & $.54^{* * *}$ & & \\
7-ESE-EM & 5.79 & 1.21 & $.23^{* * *}$ & .11 & $.43^{* * *}$ & $.45^{* * *}$ & $.40^{* * *}$ & $.54 * * *$ & \\
8-ESE-HCC & 5.94 & 0.94 & $.32^{* * *}$ & $.16^{* *}$ & $.63^{* * * *}$ & $.61^{* * *}$ & $.57^{* * *}$ & $.56^{* * *}$ & $.63^{* * *}$ \\
\hline$* * *=\mathrm{p} \leq 0,001$ & $* *=\mathrm{p} \leq 0,01$ & $*=\mathrm{p} \leq 0,05$ & & & & &
\end{tabular}

On constate d'abord que tous les indices d'ajustement des modèles testés sont très acceptables, ayant un RMSEA de 0,0417, un SRMR de 0,0604, un CFI de 0,9897 et un NFI de 0,9652 (voir le Tableau 4). Un $\chi^{2}$ est même non significatif (modèle testant l'opportunity recognition (OR)). Trois des six sous-échelles d'auto-efficacité entrepreneuriale sont significativement influencées par les fonctions du mentor, soit «définir la finalité fondamentale » (DCP), « reconnaître les opportunités » (OR) et « compétence humaine et conceptuelle» (HCC). Les autres sous-échelles d'auto-efficacité sont non significatives. Il convient aussi de souligner la pertinence de l'orientation dans un but d'apprentissage (LGO) comme variable de contrôle dans tous ces modèles. Cette variable influence significativement le développement des sous-échelles d'autoefficacité entrepreneuriale ainsi que les fonctions du mentor. L'hypothèse 1 doit être acceptée partiellement et les hypothèses 2 et 3 doivent être acceptées totalement.

\section{DISCUSSION}

Les résultats précédents viennent d'abord confirmer l'influence du mentorat dans le développement du sentiment d'auto-efficacité entrepreneuriale, mais pas sur l'ensemble des sous-mesures. Parmi les sous-mesure significatives, celle relative à la définition de la finalité fondamentale (DCP) de l'entreprise est une autre manière de parler de la clarification de la vision de l'entrepreneur, au sens de Filion (1991). En effet, deux des trois items de la mesure font explicitement référence à la vision. Il s'agit d'ailleurs d'une retombée identifiée dans une

\footnotetext{
${ }^{6}$ Les scores des construits ont été calculés à partir des items amalgamés pour en déterminer les moyennes, écarts-type et corrélations.

${ }^{7}$ Les corrélations ont été permises entre les erreurs de mesure des items suivants : LGO7 et LGO8, LGO7 et LGO5, LGO4 et LGO8, LGO3 et LGO5, LGO5 et LGO8, FCMCFR4 et FCMGUI2 ainsi que FCMSI1 et FCMREF1.

${ }^{8} \mathrm{LGO}=$ Orientation dans un but d'apprentissage
} 
recherche précédente (St-Jean, 2008). Ces résultats laissent croire qu'à défaut d'être en mesure de pouvoir évaluer de manière objective la clarification de la vision d'affaires de l'entrepreneur, on sait maintenant que le mentorat le conduit à se sentir davantage efficace à ce niveau. Certains auteurs soulignent que la discussion avec un mentor amène à clarifier la vision initiale, proposée lors du démarrage de l'entreprise, et amène parfois de nouvelles perspectives (Gravells, 2006; Pitts, 2008; Thompson et Downing, 2007). Les résultats le laissent présager.

Tableau 4. Coefficients standardisés et indices d'ajustement des modèles testés.

\begin{tabular}{lcccccc}
\hline & DCP & CUC & OR & FP & EM & HCC \\
\hline LGO & $0,44^{* * *}$ & $0,44^{* * *}$ & $0,32^{* * *}$ & $0,28^{* * *}$ & $0,18^{*}$ & $0,37^{* * *}$ \\
Fonctions & $0,22^{* *}$ & 0,03 & $0,18^{*}$ & 0,15 & 0,10 & $0,15^{*}$ \\
LGO->Fct & $0,16^{*}$ & $0,16^{*}$ & $0,16^{*}$ & $0,16^{*}$ & $0,16^{*}$ & $0,16^{*}$ \\
\hline$\chi^{2}$ & $227,87^{* * *}$ & $220,66^{* * *}$ & 184,33 & $223,04^{* * *}$ & $209,98^{* *}$ & $202,92^{* *}$ \\
d.l. & 160 & 160 & 160 & 160 & 160 & 160 \\
RMSEA & 0,0417 & 0,0394 & 0,0250 & 0,0402 & 0,0358 & 0,0332 \\
SRMR & 0,0583 & 0,0604 & 0,0512 & 0,0574 & 0,0556 & 0,0562 \\
CFI & 0,9897 & 0,9905 & 0,9962 & 0,9899 & 0,9921 & 0,9934 \\
NFI & 0,9665 & 0,9666 & 0,9720 & 0,9652 & 0,9907 & 0,9699 \\
\hline
\end{tabular}

$* * *=\mathrm{p} \leq 0,001 \quad * *=\mathrm{p} \leq 0,01 \quad *=\mathrm{p} \leq 0,05$

L'autre sous-mesure significative concerne l'auto-efficacité entrepreneuriale à reconnaître des opportunités (OR). Comme le laissent penser les résultats, l'entrepreneur novice se sent davantage compétent à identifier des opportunités d'affaires grâce aux conseils et encouragements du mentor, par exemple. Ces résultats viennent donner du crédit à des recherches précédentes qui suggéraient l'influence du mentor dans l'identification d'opportunités (Gordon, 2007; Ozgen et Baron, 2007). Le mentor suscite la génération de nouvelles options pour l'entreprise de l'entrepreneur novice (Gravells, 2006). L'entrepreneur qui se limite aux connaissances basées sur ses expériences personnelles finit par être limité dans la reconnaissance d'opportunités, mais il peut outrepasser ce seuil par la discussion avec des mentors (Ucbasaran et al., 2009). Qui plus est, tel que le proposait Krueger (2000), c'est en contribuant au développement du sentiment d'auto-efficacité de l'entrepreneur que son mentor pourrait lui permettre d'identifier davantage d'opportunités Les résultats obtenus ici démontrent la justesse de la proposition de Krueger (2000) et permettent d'envisager que le mentorat pourrait effectivement influencer la compétence de l'entrepreneur à reconnaître des opportunités.

La dernière sous-mesure d'auto-efficacité entrepreneuriale qui est développée par le mentorat concerne la compétence humaine et conceptuelle (HCC). Les items font explicitement référence à la supervision, à la direction, à l'influence et à la capacité à motiver les employés, ce qui ramène aux qualités de leader de l'entrepreneur. Ici aussi, à défaut d'avoir une mesure « objective » pour vérifier l'acquisition de certaines compétences, notamment celles relatives au leadership du nouveau dirigeant, on peut constater que le mentorat augmente l'auto-efficacité relative au leadership. D'autres recherches ont mis en évidence l'influence du mentorat dans le développement de certaines compétences spécifiques (Deakins et al., 1998; Lane, 2003). On suggérait d'ailleurs de mettre en relation des entrepreneurs avec des mentors pour leur permettre de développer des habiletés de leadership spécifiques au développement de leur entreprise (Sullivan, 2000). Aussi, des jeunes ayant participé à des cours d'entrepreneuriat où ils étaient jumelés à des mentors affirment majoritairement avoir augmenté leurs compétences de leader (Hernandez et Newman, 2006). Dans la présente recherche, il est démontré que le mentorat influence l'auto-efficacité, et non la compétence elle-même, mais il demeure néanmoins probable que certaines compétences puissent avoir été développées, bien qu'il soit impossible de le prouver empiriquement par ces analyses. 
Si cette recherche a pu illustrer l'influence du mentorat sur certaines mesures d'auto-efficacité, comme il l'a été suggéré précédemment (Nandram, 2003), d'autres mesures se sont avérées non significatives. L'auto-efficacité entrepreneuriale à faire face à des défis inattendus (CUC), à planifier formellement (FP) et à gérer financièrement l'entreprise (EM) ne sont pas influencées par le mentorat. Pour la mesure de planification formelle, il est tout à fait probable que la petite taille de l'entreprise des mentorés ne nécessite pas une grande planification formelle des activités et, conséquemment, il est probable que cet aspect n'ait pas été travaillé par le mentor. En effet, rappelons que le chiffre d'affaires de l'entreprise des mentorés est majoritairement inférieur à $100000 \$$ annuellement $(62,8 \%)$ et pour $88,9 \%$ d'entre eux, il est inférieur à $500000 \$$, avec une moyenne de 4,48 employés. Au niveau de l'auto-efficacité à gérer financièrement l'entreprise, il est aussi possible que le mentorat ne puisse pas être utile. Le réseau de mentorat d'affaires suggère aux coordonnateurs des cellules d'éviter de jumeler les novices avec des mentors " experts », par exemple en comptabilité ou en finance, avec l'objectif d'éviter de faire évoluer la relation vers du coaching, c'est-à-dire dans le but de développer des compétences spécifiques, plutôt que générales. Le mentor n'est donc pas là pour aider l'entrepreneur novice à développer sa compétence à mieux gérer l'entreprise financièrement, ne contribuant pas à influencer l'auto-efficacité, ni même le développement de compétences, à ce niveau.

L'orientation dans un but d'apprentissage a un effet sur les fonctions du mentor, tel qu'il l'a été démontré dans le contexte du mentorat dans la grande organisation (Egan, 2005; Godshalk et Sosik, 2003). Qui plus est, cette variable s'est avérée significative dans le développement de l'auto-efficacité. Ces résultats viennent confirmer l'importance de considérer l'orientation dans un but d'apprentissage dans le développement du sentiment d'auto-efficacité, tel qu'il l'a été démontré dans d'autres champs de recherche (Bell et Kozlowski, 2002; Phillips et Gully, 1997). Soulignons que contrairement aux recherches précédentes, les mesures d'auto-efficacité sont contextualisées à l'entrepreneuriat. Cela démontre l'intérêt de considérer l'effet de l'orientation dans un but d'apprentissage de l'entrepreneur pour comprendre le développement de certaines retombées, notamment de l'auto-efficacité, et ce, malgré un échantillon qui illustrait peu de variance sur cette variable. Rappelons ici qu'il est impossible de savoir si ce sont les répondants qui sont davantage orientés dans le but d'apprendre que les entrepreneurs novices qui ne choisissent pas ce mode d'accompagnement, ou bien si ce sont les entrepreneurs, en général, qui sont plus orientés dans un but d'apprentissage que la population en général. Tout ce que l'on constate, c'est que cette variable est définitivement médiatrice dans la relation entre le mentorat reçu et l'auto-efficacité entrepreneuriale. Il serait quand même utile, pour les recherches futures, de considérer l'orientation dans un but d'apprentissage et son influence dans le développement de l'auto-efficacité entrepreneuriale dans d'autres contextes que le mentorat. Dans une perspective plus large, il serait aussi intéressant d'investiguer le rôle de cette variable dans la capacité de l'entrepreneur à apprendre des situations vécues dans son entreprise, à en tirer des leçons et, conséquemment, à mieux performer.

D'autres recherches sont aussi envisagées dans le but de compléter cette analyse. Si l'on constate que le mentorat reçu, tel que mesuré par les fonctions du mentor, est en relation avec le développement de certaines mesures d'auto-efficacité entrepreneuriale, il faut néanmoins garder à l'esprit qu'il s'agit de la perception des fonctions déployées et ce, du point de vue du mentoré exclusivement. En aucun cas le mentor n'a été questionné, ce qui ne donne qu'un portrait partiel de la réalité. Il pourrait être intéressant de voir dans quelle mesure les fonctions du mentor, tel que ce dernier les perçoit, influencent le développement de l'auto-efficacité entrepreneuriale du mentoré. Également, il faut souligner que les fonctions du mentor ont été traitées dans cette recherche d'un seul bloc, en tant que mesure de mentorat reçu (et tel que perçu par le mentoré). Bien que cela permette de "quantifier» le mentorat, la perte d'information engendrée empêche de voir l'apport de chacune des fonctions, d'une part, et suggère des rôles plutôt uniformes chez 
les mentors, n'ayant que l'amplitude du mentorat desservi comme variance, d'autre part, ce qui ne représente certes pas la réalité beaucoup plus complexe.

Une autre limite importante à souligner concerne la modélisation restrictive utilisée pour cette recherche. Évidemment, même si nous savons maintenant que le mentorat influence l'autoefficacité entrepreneuriale, plusieurs autres processus permettent de développer cette dimension. On peut penser aux formations reçues, aux encouragements des conjoints, parents et amis, aux expériences précédentes en entrepreneuriat, sans compter plusieurs autres particularités psychologiques ou physiologiques individuelles. Nous avons retenu l'orientation dans un but d'apprentissage puisque cette dimension psychologique avait un effet dans le contexte du mentorat (p. ex. Godshalk et Sosik, 2003) mais d'autres variables pourraient également compter. N'ayant pas considéré une panoplie de variable ayant un effet confirmé dans le développement de l'auto-efficacité entrepreneuriale dans les analyses effectuées ici, nous ne pouvons pas pondérer l'effet du mentorat par rapport à d'autres variables pouvant être associés aux quatre processus du développement de l'auto-efficacité de Bandura (1986).

De plus, la nature transversale de l'étude apporte une certaine limite à la démonstration, qui devrait être amélioré par une recherche longitudinale dans le cadre d'une recherche future, qui pourrait mesurer l'auto-efficacité «avant» le jumelage et une fois la relation terminée. En outre, un groupe de contrôle de novices n'ayant pas de mentor pourrait permettre de démontrer, hors de tout doute, l'effet du mentorat sur le développement de l'auto-efficacité entrepreneuriale pour un groupe comparable d'entrepreneurs. Ces pistes ne sont que quelques-unes de celles envisageables dans le futur pour compléter les résultats obtenus et poursuivre l'investigation sur ces différentes dimensions.

\section{CONCLUSION}

Bien entendu, cette recherche a permis de démontrer l'effet du mentorat dans le développement de l'auto-efficacité entrepreneuriale chez les novices. De manière concomitante, les entrepreneurs novices faisant preuve d'une orientation élevée dans un but d'apprentissage vont également développer davantage d'auto-efficacité entrepreneuriale. Malgré ces résultats encourageants, il reste de nombreuses analyses complémentaires pour comprendre comment le mentor parvient à développer ce sentiment chez le mentoré. En effet, nous ne savons que très peu de choses à propos des profils de mentors permettant de produire un maximum de retombées chez le novice. Une telle connaissance serait particulièrement utile pour les organismes publics qui interviennent dans des programmes de mentorat. Bien que ceux-ci puissent maintenant apprécier davantage cette forme d'accompagnement, ils doivent aussi garder à l'esprit que d'autres retombées sont aussi possibles pour les mentorés. Cela invite à poursuivre les recherches dans ce secteur prometteur.

\section{RÉFÉRENCES}

ANNA, A. L., CHANDLER, G. N., JANSEN, E. et MERO, N. P. (2000), " Women business owners in traditional and non-traditional industries ", Journal of Business Venturing, vol. $15, \mathrm{n}^{\mathrm{o}} 3$, p. 279-303.

ARMSTRONG, J. S. et OVERTON, T. S. (1977), " Estimating Nonresponse Bias in Mail Surveys ", Journal of Marketing Research, vol. 14, n 3, p. 396-402.

BALDWIN, J., GRAY, T., JOHNSON, J., PROCTOR, J., RAFIQUZZAMAN, M. et SABOURIN, D. (1997), Les faillites d'entreprise au Canada, Rapport no ${ }^{\circ}$ 61-525-XIF, Statistique Canada, Ottawa.

BANDURA, A. (1986), Social Foundations of Thought and Action - A Social Cognitive Theory, Englewood Cliffs, N.J., Prentice-Hall, 617 p. 
BANDURA, A. (1997), Self-efficacy : the exercise of control, New York, W.H. Freeman, 604 p.

BARBOSA, S. D., GERHARDT, M. W. et KICKUL, J. R. (2007), " The Role of Cognitive Style and Risk Preference on Entrepreneurial Self-Efficacy and Entrepreneurial Intentions ", Journal of Leadership \& Organizational Studies, vol. 13, nº 4, p. 86-104.

BELL, B. S. et KOZLOWSKI, S. W. J. (2002), " Goal orientation and ability: Interactive effects on self-efficacy, performance, and knowledge ", Journal of Applied Psychology, vol. 87, no 3, p. 497-505.

BISK, L. (2002), " Formal entrepreneurial mentoring: the efficacy of third party managed programs ", Career Development International, vol. 7, no 5, p. 262-270.

BOUQUILLON, E. A., SOSIK, J. J. et LEE, D. (2005), " 'It's only a phase': examining trust, identification and mentoring functions received accross the mentoring phases ", Mentoring \& Tutoring, vol. 13, $\mathrm{n}^{\mathrm{o}} 2$, p. 239-258.

BUTTON, S. B., MATHIEU, J. E. et ZAJAC, D. M. (1996), " Goal Orientation in Organizational Research: A Conceptual and Empirical Foundation ", Organizational Behavior and Human Decision Processes, vol. 67, $\mathrm{n}^{\mathrm{o}}$ 1, p. 26-48.

CULL, J. (2006), " Mentoring Young Entrepreneurs: What Leads to Success? ", International Journal of Evidence Based Coaching and Mentoring, vol. 4, n 2, p. 8-18.

D'ABATE, C. P., EDDY, E. R. et TANNENBAUM, S. I. (2003), " What's in a Name? A Literature-Based Approach to Understanding Mentoring, Coaching, and Other Constructs That Describe Developmental Interactions ", Human Resource Development Review, vol. 2, no 4, p. 360-384.

DE NOBLE, A. F., JUNG, D. et EHRLICH, S. B. (1999), « Entrepreneurial Self-Efficacy: The Development of a Measure and Its Relationship to Entrepreneurial Action », Babson: Babson College. Frontiers of Entrepreneurship Research Accès le 4 juillet 2007. http://www.babson.edu/entrep/fer/papers99/I/I C/IC.html.

DEAKINS, D., GRAHAM, L., SULLIVAN, R. et WHITTAM, G. (1998), " New venture support: an analysis of mentoring support for new and early stage entrepreneurs ", Journal of Small Business and Enterprise Development, vol. 5, $\mathrm{n}^{\mathrm{o}}$ 2, p. 151-161.

DESCHAMPS, B., FATIEN, P. et GEINDRE, S. (2010), " Accompagner le repreneur d'entreprise : conduire, escorter mais aussi guider ", Gestion 2000, vol. 27, nº 3, p. 7788.

DWECK, C. S. (1986), " Motivational processes affection learning ", American Psychologist, vol. 41, n ${ }^{\circ} 10$, p. 1040-1048.

EGAN, T. M. (2005), " The Impact of Learning Goal Orientation Similarity on Formal Mentoring Relationship Outcomes ", Advances in Developing Human Resources, vol. 7, $\mathrm{n}^{\mathrm{o}} 4$, p. 489-504.

ENSHER, E. A., MURPHY, S. E. et VANCE, C. M. (2000), " Mentoring and self-management career strategies for entrepreneurs ", International Journal of Entrepreneurship and Innovation, vol. $1, \mathrm{n}^{\circ} 2$, p. 99-108.

FESTERVAND, T. A. et FORREST, J. E. (1991), « Small Business Failures: A Framework for Analysis ». Small Business Institute Director's Association Conference, Orlando, FL, p. $1-14$.

FILION, L.-J. (1991), " Vision and relation: Elements for an entrepreneurial metamodel ", International Small Business Journal, vol. 9, n 2, p. 26-40.

GASKILL, L. R., VAN AUKEN, H. E. et MANNING, R. A. (1993), " A Factor Analytic Study of the Perceived Causes of Small Business Failure ", Journal of Small Business Management, vol. 31, no 4, p. 18-31.

GIBSON, S. K. (2005), " Whose Best Interests Are Served? The Distinction Between Mentoring and Support ", Advances in Developing Human Resources, vol. 7, n 4, p. 470-488.

GODSHALK, V. M. et SOSIK, J. J. (2003), " Aiming for career success: The role of learning goal orientation in mentoring relationships ", Journal of Vocational Behavior, vol. 63, $\mathrm{n}^{\mathrm{o}} 3$, p. 417-437. 
GORDON, S. R. (2007), " Interpersonal trust, vigilance and social networks roles in the process of entrepreneurial opportunity recognition ", International Journal of Entrepreneurship and Small Business, vol. 4, $\mathrm{n}^{\circ}$ 5, p. 564-585.

GRAVELLS, J. (2006), " Mentoring start-up entrepreneurs in the East Midlands Troubleshooters and Trusted Friends ", The International Journal of Mentoring and Coaching, vol. $4, \mathrm{n}^{\mathrm{o}} 2$.

HAGGARD, D. L., DOUGHERTY, T. W., TURBAN, D. B. et WILBANKS, J. E. (2011), " Who Is a Mentor? A Review of Evolving Definitions and Implications for Research ", Journal of Management, vol. 37, $\mathrm{n}^{\circ}$ 1, p. 280-304.

HERNANDEZ, S. A. et NEWMAN, C. M. (2006), " Minding Our Business: A model of service-learning in entrepreneurship ", Journal of Entrepreneurship Education, vol. 9, $\mathrm{n}^{\mathrm{o}} 1$, p. 53-75.

HMIELESKI, K. M. et CORBETT, A. C. (2008), " The contrasting interaction effects of improvisational behavior with entrepreneurial self-efficacy on new venture performance and entrepreneur work satisfaction ", Journal of Business Venturing, vol. 23, n $\mathrm{n}^{\mathrm{0}}$ 4, p. 482-496.

HOMÈRE (2001), Odyssée (V. Bédard et E. Cantarella, trad.), Paris, Les Belles Lettres.

JOHANNISSON, B. (1991), " University training for entrepreneurship: a Swedish approach ", Entrepreneurship and Regional Development, vol. 3, $\mathrm{n}^{\mathrm{o}}$ 1, p. 67-82.

JÖRESKOG, K. et SÖRBOM, D. (2002), PRELIS 2: User's Reference Guide (3 éd.), Lincolnwood, IL, SSI inc., $224 \mathrm{p}$.

KANFER, R. (1990), " Motivation and individual differences in learning: An integration of developmental, differential and cognitive perspectives ", Learning and Individual Differences, vol. 2, p. 221-239.

KIM, S. (2007), " Learning goal orientation, formal mentoring, and leadership competence in HRD: A conceptual model ", Journal of European Industrial Training, vol. 31, n 3, p. 181-194.

KRAM, K. E. (1985), Mentoring at Work : Developmental Relationships in Organizational Life Glenview, Ill., Scott Foresman, 252 p.

KRUEGER, N. F. J. (2000), " The Cognitive Infrastructure of Opportunity Emergence ", Entrepreneurship: Theory \& Practice, vol. 24, $\mathrm{n}^{\circ} 3$, p. 5-23.

LANE, D. C. (2003), « Mentoring and Link Teacher Roles within Young Enterprise ». ICSB $48^{\text {th }}$ World Conference, North Ireland, June, 15-18.

LANKAU, M. J. et SCANDURA, T. A. (2002), " An investigation of personal learning in mentoring relationships: content, antecedents, and consequences ", Academy of Management Journal, vol. 45, no 4, p. 779-790.

MACCALLUM, R. C., BROWNE, M. W. et SUGAWARA, H. M. (1996), " Power Analysis and Determination of Sample Size for Covariance Structure Modeling ", Psychological Methods, vol. 1, no 2, p. 130-149.

MAURER, T. J. (2001), " Career-relevant learning and development, worker age, and beliefs about self-efficacy for development ", Journal of Management, vol. 27, $\mathrm{n}^{\circ}$ 2, p. 123140.

MCGREGOR, J. et TWEED, D. (2002), " Profiling a New Generation of Female Small Business Owners in New Zealand: Networking, Mentoring and Growth ", Gender, Work and Organization, vol. 9, n ${ }^{\circ}$ 4, p. 420-438.

NANDRAM, S. S. (2003), "Entrepreneurs' Need For Mentoring And Their Individual Differences ». ICSB 48 ${ }^{\text {th }}$ World Conference, North Ireland, June, 15-18.

OZGEN, E. et BARON, R. A. (2007), " Social sources of information in opportunity recognition: Effects of mentors, industry networks, and professional forums ", Journal of Business Venturing, vol. 22, $\mathrm{n}^{\circ}$ 2, p. 174-192.

PAUL, M. (2004), L'accompagnement : une posture professionnelle spécifique, Paris, L'Harmattan, $356 \mathrm{p}$. 
PELLEGRINI, E. K. et SCANDURA, T. A. (2005), " Construct equivalence across groups: an unexplored issue in mentoring research ", Educational and Psychological Measurement, vol. $65, \mathrm{n}^{\mathrm{o}} 2$, p. 323-335.

PHILLIPS, J. M. et GULLY, S. M. (1997), " Role of goal orientation, ability, need for achievement, and locus of control in the self-efficacy and goal-setting process ", Journal of Applied Psychology, vol. 82, $\mathrm{n}^{\circ}$ 5, p. 792-802.

PITTS, G. (2008), " Life as an entrepreneur: leadership and learning ", Development and Learning in Organizations, vol. 22, $\mathrm{n}^{\circ} 3$, p. 16-17.

ROBERTSON, I. T. et SADRI, G. (1993), " Managerial self-efficacy and managerial performance ", British Journal of Management, vol. 4, $n^{\circ}$ 1, p. 37-46.

SCANDURA, T. A. et WILLIAMS, E. A. (2001), " An Investigation of the Moderating Effects of Gender on the Relationships between Mentorship Initiation and Protégé Perceptions of Mentoring Functions ", Journal of Vocational Behavior, vol. 59, nº 3, p. 342-363.

SCHERER, R. F., ADAMS, J. S., CARLEY, S. S. et WIEBE, F. A. (1989), " Role Model Performance Effects on Development of Entrepreneurial Career Preference ", Entrepreneurship Theory and Practice, vol. 13, $\mathrm{n}^{\circ}$ 3, p. 53-71.

ST-JEAN, E. (2008), " La formation destinée à l'entrepreneur novice : exploration des possibilités offertes par le mentorat ", Revue de l'Entrepreneuriat, vol. 7, no 1, p. 1-22.

ST-JEAN, E. (2010), " Les fonctions du mentor de l'entrepreneur novice ", Revue de l'entrepreneuriat, vol. $9, \mathrm{n}^{\circ} 2$.

ST-JEAN, E. et AUDET, J. (Under press), " The Role of Mentoring in the Learning Development of the Novice Entrepreneur ", International Entrepreneurship and Management Journal.

ST-JEAN, É., AUDET, J. et BOUCHER, N. (2007), « L'apprentissage de l'entrepreneur réalisé dans le cadre d'une relation de mentorat : exploration des possibilités ». 24 Congrès annuel du CCPME/CCSBE, Kelowna, C.B., $1^{\mathrm{e}}$ au 3 novembre.

SULLIVAN, R. (2000), " Entrepreneurial learning and mentoring ", International Journal of Entrepreneurial Behaviour \& Research, vol. 6, n 3, p. 160-175.

TABACHNICK, B. G. et FIDELL, L. S. (2007), Using Multivariate Statistics (5 éd.), Boston, MA, Allyn \& Bacon, 980 p.

TEPPER, K., SHAFFER, B. C. et TEPPER, B. J. (1996), " Latent structure of mentoring function scales ", Educational and Psychological Measurement, vol. 56, n ${ }^{\circ}$ 5, p. 848857.

THOMPSON, J. et DOWNING, R. (2007), " The entrepreneur enabler: identifying and supporting those with potential ", Journal of Small Business and Enterprise Development, vol. 14, n ${ }^{\circ} 3$, p. 528-544.

UCBASARAN, D., WESTHEAD, P. et WRIGHT, M. (2009), " The extent and nature of opportunity identification by experienced entrepreneurs ", Journal of Business Venturing, vol. 24, $\mathrm{n}^{\mathrm{o}}$ 2, p. 99-115.

WANBERG, C. R., WELSH, E. T. et HEZLETT, S. A. (2003), " Mentoring Research: A Review and Dynamic Process Model », dans J. J. Martocchio et G. R. Ferris (dir.), Research in Personnel and Human Resources Management, Oxford, U.K., Elsevier Science Ltd. Vol. 22, p. 39-124.

WATERS, L., MCCABE, M., KIELLERUP, D. et KIELLERUP, S. (2002), " The role of formal mentoring on business success and self-esteem in participants of a new business start-up program ", Journal of Business and Psychology, vol. 17, $\mathrm{n}^{\circ}$ 1, p. 107-121.

WIKHOLM, J., HENNINGSON, T. et HULTMAN, C. M. (2005), « Demand of mentoring among new starters ». ICSB $50^{\text {th }}$ World Conference, Washington, DC.

WILSON, F., KICKUL, J. R. et MARLINO, D. (2007), " Gender, Entrepreneurial SelfEfficacy, and Entrepreneurial Career Intentions: Implications for Entrepreneurship Education ", Entrepreneurship Theory and Practice, vol. 31, n 3, p. 387-407. 
ZIMMERMAN, B. et MARTINEZ-PONS, M. (1990), " Student differences in self-regulated learning: Relating grade, sex, and giftedness to self-efficacy and strategy use ", Journal of Educational Psychology, vol. 82, nº 1, p. 51-59. 